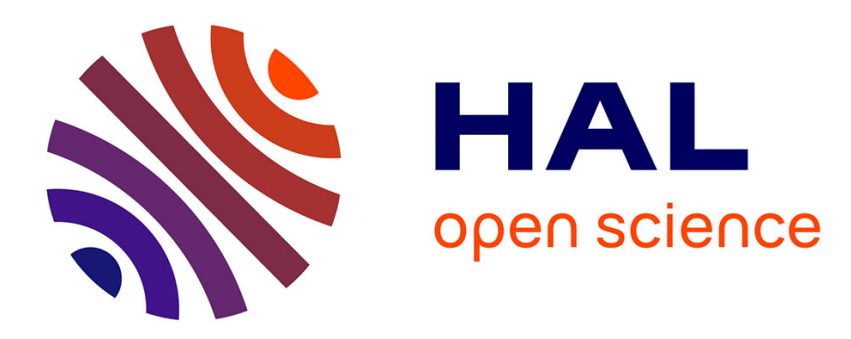

\title{
A mosquito bite against the enactive approach to bodily experiences
}

Frédérique de Vignemont

\section{To cite this version:}

Frédérique de Vignemont. A mosquito bite against the enactive approach to bodily experiences. Journal of Philosophy, 2011, pp.1-23. ijn_00512316

\section{HAL Id: ijn_00512316 \\ https://hal.science/ijn_00512316}

Submitted on 30 Aug 2010

HAL is a multi-disciplinary open access archive for the deposit and dissemination of scientific research documents, whether they are published or not. The documents may come from teaching and research institutions in France or abroad, or from public or private research centers.
L'archive ouverte pluridisciplinaire HAL, est destinée au dépôt et à la diffusion de documents scientifiques de niveau recherche, publiés ou non, émanant des établissements d'enseignement et de recherche français ou étrangers, des laboratoires publics ou privés. 


\section{A mosquito bite against the enactive approach to bodily experiences}

Frédérique de Vignemont

Transitions

CNRS - NYU

Correspondence should be addressed to Frederique de Vignemont, New York University, Department of philosophy, 5 Washington Place, New York 10003 NY, email: fdv208@nyu.edu 
"But, I don't understand that. You put something there; I do not feel anything and yet I got there with my finger. How does that happen?" ${ }^{1}$

\section{Introduction}

The enactive approach rejects the classical computational dichotomy between perception and action. ${ }^{2}$ Perception is not merely a means to action and action a means to perception. Instead, the proponents of the enactive approach argue for a stronger relation of constitutive interdependence. On this view, perceptual content constitutively depends on law-like relationships that hold between sensory input and motor output. Hence, perceptual experiences are said to be inseparable from the perceiver's bodily activities.

Touch is taken by the enactive approach as paradigmatic of perception because the content of one's tactile perception of the shape of an object is said to be constitutively determined by the action of exploring it with one's fingers.

"All perception is touch-like in this way: perceptual experience acquires content thanks to our possession of bodily skills. What we perceive is determined by what we do (or what we know how to do).” Noë $(2004$, p. 1)

"Touch acquires spatial content — comes to represent spatial qualities — thanks to the ways touch is linked to movement and to our implicit understanding of the

\footnotetext{
${ }^{1}$ Report of a patient suffering from numbsense in J. Paillard, F. Michel and G. Stelmach, "Localization without content: a tactile analogue of 'blind sight"', Archives of-Neurology, 40 (1983), p.550.

${ }^{2}$ This view has been defended by, among others, Susan Hurley in Consciousness in Action (Cambridge: Harvard, 1998); Kevin O'Regan and Alva Noë, "A sensorimotor account of vision and visual consciousness", Behavioral and Brain Sciences. 24, 5 (2001): 939-101; Alva Noë, Action in Perception (Cambridge: MIT Press, 2004). Evan Thompson, "Sensorimotor subjectivity and the enactive approach to experience", Phenomenology and the Cognitive Sciences. 4 (2005): 407-427.
} 
relevant tactile-motor dependencies governing our interaction with objects.” idem, p. 205

Although it is vision that has received the most attention, it seems indeed that the enactive approach is more likely to be true of tactile experiences than those of any other senses (e.g., gustatory experiences). For instance, in haptic touch, the tactile system is not a passive recipient of bodily information, and consists in exploratory activities. However, what may be true for haptic touch, which is intrinsically active, may be more controversial for passive touch (e.g., the sensation of a mosquito on your skin). If touch is to be the model for all perception, or at least the less controversial application of the enactive approach, then it is worth a more thorough examination.

After drawing the agenda for an enactive view of tactile experiences, I shall highlight the difficulties that it has to face, both conceptual and empirical. I shall conclude that what we do does not determine what we feel.

\section{The agenda for an enactive approach to tactile experiences}

What is at the core of the enactive theories is the dynamic constitutive relationship between sensory input and motor output. On this view, we are able to keep track of the interdependence between the sensory input and the motor output, this ability being grounded in the procedural knowledge of how the way one moves affects the sensory signals that one receives (or how the movement of objects will affect those signals). In this sense, Hurley talked of "active perception" and Noë describes perception as an "embodied activity".

One should, however, acknowledge the diversity of views that enactivism includes. There is indeed little agreement among the proponents of the enactive approach beyond the claim of the interdependence of perception and action. One noteworthy difference is the variety of 
explananda of the enactive views. There are at least three aspects that are thought to require an account in enactive terms: (i) the individuation of perceptual modalities (e.g. sight vs. audition); (ii) perceptual phenomenology (i.e. what it is like to perceive) and (iii) perceptual content (i.e. representational content). First, it has been argued that each sensory modality depends on its own specific patterns of dynamic interdependence between sensory stimulation and movements. The set of patterns differs depending on whether one is seeing or touching an object for instance, and as such it would individuate the various sensory modalities, e.g. distinguish sight from audition. Second, some versions of the enactive approach claim to account for consciousness, and more precisely for the qualitative phenomenology of perceptual experiences. For instance, what it is like to see a red tomato would consist in the procedural knowledge of the sensory effects of one's movements relative to the light. Finally, most enactive theories aim at explaining the intentional content of perceptual experiences, and more particularly how perceptual experiences represent spatial properties. Seeing that the table is round is to know that it looks more or less elliptical depending on where one is relative to the table.

Here I am not interested in the idiosyncrasies of the various enactive approaches. ${ }^{3}$ Rather I shall propose different ways to spell out the enactive view of tactile experiences and focus on the most plausible ones. Following the distinctions above, an enactivist for tactile experiences can pursue the following aims: (i) to individuate the tactile modality (e.g., a tactile experience, by contrast with a visual one); (ii) to explain the phenomenology of tactile sensations (e.g., the conscious sensation of pressure on my skin); (iii) to explain the spatial content of tactile experiences (e.g., the sensation that there is an object touching me on my palm that is round). In the last case, the enactivist shall have to take into account the duality of the content of

\footnotetext{
${ }^{3}$ For a review of the differences between versions of the enactive theory of vision, see Gangopadhyay and Kiverstein, "Enactivism and the Unity of Perception and Action", Topoi, 28 (2009): 63-73.
} 
tactile experiences. By contrast with other sensory modalities, touch is both about the external world with which one is in contact and about one's body that is in contact with the external world. Hence, an enactive account of tactile experiences must accommodate both exteroceptive content and interoceptive content.

To add to the complexity, touch is a very comprehensive term, so much so that one may even deny that it refers to a unique sensory modality. On the one hand, there is active touch. It is primarily defined by movements of the body part that is in contact with an object. The movements can be of three types. Some are merely passive movements (e.g., your hand is moved by another individual). Some are exploratory movements, voluntarily initiated by the perceiver (e.g., you follow the contours of the object). Some are effortful movements (e.g., you lift and manipulate an object with muscular effort). On the other hand, there is passive touch, which is merely cutaneous. Here again, one may distinguish between static passive touch (e.g., the pressure of the cat on your knees) and kinematic passive touch (e.g., the motion of the mosquito on your skin).

In virtue of their interoceptive content, tactile experiences are partly bodily experiences, or in more enactive terms, embodied. However, what matters for the enactive approach is not so much the embodiment of perception as its intrinsic relation with action. And it is controversial whether tactile experiences consist in an embodied activity. True, in haptic touch, I move my body to retrieve information about an object when needed and the movements of my hand over a surface induce shifting sensations. But can one generalize the enactive account of haptic experiences to any kind of tactile experiences, and more generally to bodily experiences? How can the so-called passive touch be active?

Here I shall attempt to apply the enactive approach to instantaneous passive touch, which seems to be the most challenging case. Let us imagine that I consciously feel a short nonpainful tap on my knee. The content of my experience is not very rich. I may not even be able 
to know what touched me. Yet, I feel that I was briefly touched on my knee, and not anywhere else. What grounds my phenomenology and the spatial content of my tactile experience? If one succeeds in offering an enactive account of such an impoverished tactile experience, then there is a good chance that one can offer a more general enactive account of bodily experiences. Let us see now how this might work. We shall see that the task is not easy.

\section{Tactile-motor expectations}

Visual experiences are said to depend on eye movements, and haptic touch on exploratory hand movements. But I do not need to move to have a tactile experience, as is the case in passive touch. Yet, a proponent of the enactive approach may claim that even in passive touch, one masters the procedural knowledge of the tactile consequences of potential movements (i.e. sensorimotor contingencies). What matters is not the bodily exercise of procedural knowledge, but its mere possession. For instance, itching sensations would consist in the knowledge that if one scratches where one has been bitten by the mosquito, the stimulation would decrease. One does not need to scratch one's leg, it suffices that one knows the consequences of doing so. The same could be said for tactile experiences. What would constitute tactile experiences is not what you do, but what you expect to feel if you do it. But what kind of procedural knowledge is constitutive of passive touch?

Let me start with two possible tactile-motor expectations. ${ }^{4}$

(1) If I remove my knee from the object it is in contact with, I shall stop receiving tactile signals.

(2) If I remove the object with my hand, I shall stop receiving tactile signals.

\footnotetext{
${ }^{4}$ Many questions have been raised on the specific nature of sensorimotor knowledge (for full review in the case of visual experiences, see the commentaries in O'Regan \& Noë, 2001). Here I shall not insist on these general difficulties about the enactive account, but focus on specific problems raised by tactile-motor knowledge.
} 
As basic and uncontroversial as these tactile-motor expectations are, they raise several worries. The closest equivalent in vision would be the expectation that if I close my eyes, I will no longer receive visual signals. Nobody in the computational community would ever deny this simple relation of causal dependence between vision and eye opening/closing, or between touch and bodily contact. However, not all causal relations between touch and action are of interest for the enactive view. According to Hurley, the interdependence of perception and action can be of two kinds. It is instrumental when action is just a means to perception. It is non-instrumental when perception wholly or partly consists in action. Only the latter kind is relevant for the enactive approach. The question then is which kind the sensorimotor expectations (1) and (2) belong to: instrumental or non-instrumental. In vision, the movement of eye opening/closing makes the visual experience possible or not, but this is a feature of any kind of visual experience. I could see a red tomato or a yellow lemon for all it matters. As such, it cannot determine the specific phenomenology and the specific content of the visual experience that results from my opening my eyes. Similarly in touch, the movement of separating the body part from the object (or getting it into contact with the object) stops the tactile experience, but it would stop any kind of tactile experience, whether I feel a rough texture or a smooth one. Hence, it cannot be an individuative condition of the phenomenology of my tactile experience nor of its descriptive content. At most, this type of sensorimotor contingency may individuate the sensory modality: vision would constitutively require stopping the flow of information by closing the eyes, and touch would constitutively require stopping the flow of information by retrieving the body part in contact with the object. Yet, it seems that most enactive proponents want more.

They may for instance argue that the tactile-motor contingency (1) can individuate at least the bodily spatial content, which is at the core of our interests. Indeed, it is only if I remove my knee from the object it is in contact with that I can expect the tactile signal to stop, and not 
any other body part. The tactile-motor contingency is body-part specific, and as such might provide the bodily spatial content. But how do I know that it is my knee that I need to move? There is a threat of circularity, which is similar to the one highlighted by Jacob (2008) in the case of vision. ${ }^{5}$ The enactive account claims that visuo-motor knowledge consists in the knowledge of how one's movements toward a visually seen object affect the way one perceives the object. But Jacob notices that in order to act upon an object, one needs first to be able to single it out, to pick it out from other objects. Hence, visuo-motor knowledge cannot allow one to individuate the relevant object. There must be another part to the story, which cannot be told in enactivist terms. Similarly, in the tactile case, it is not clear how one can individuate the body part on the basis of tactile-motor contingencies without similarly falling into circularity. The tactile-motor knowledge requires a prior and independent way of singling out the relevant body part that is in contact with the object, if one wants to avoid a circular account of the bodily spatial content.

The tactile-motor expectations (1) and (2) do not work if they are supposed to constitute the phenomenology or the content of tactile experiences. But the problem is more fundamental, and not limited to these two specific examples. To give an enactive account of the content of tactile experiences is indeed difficult, especially the interoceptive content. On some enactive views, what is constitutive of the content of perceptual experience is the procedural knowledge that gives access to objective properties based on the relation between the experience of perspectival properties and bodily activities. For instance, the table is round (i.e. objective property of the stimulus, which is independent of the perceiver), but it appears to you as being elliptical (i.e. perspectival property which is the way the stimulus appears to you). According to this enactive theory, your visual experience represents the table as round

\footnotetext{
${ }^{5}$ Pierre Jacob, "The scope and limits of the enactive approaches to visual experiences". Perception, 47 (2008): 446-461.
} 
because you know how its perspectival property would change depending on how you move relative to the object (e.g., you get closer to the table). However, it is hard to see on this account how there could be any kind of dependency between touch and action, as far as the interoceptive content of touch is concerned. When I feel the tap on my knee, it does not seem to make sense to distinguish between a perspectival bodily property and an objective bodily property, if perspectival properties depend on the spatial relation between the perceiver and the object. If the object is one's own body, then it cannot be blocked from tactile perception, nor can one change one's spatial relations to it. In other words, one cannot change the spatial conditions under which one's own body is felt. And if one cannot change the spatial conditions, then there is no corresponding tactile-motor knowledge of the way the perspectival properties would change depending on the way one moves relative to one's body. Faced with this difficulty, the proponents of the enactive view have two choices: either to restrict their account to the exteroceptive content of tactile experiences or to offer an alternative type of enactive account of the interoceptive content that differs from the one provided for the exteroceptive content.

Finally, even if the tactile-motor contingencies outlined above were satisfactory, they could not work for every kind of touch, and so they fail to satisfy the enactive agenda. For example, they cannot be easily applied to instantaneous touch. When I receive a brief tap on my knee, I cannot remove my knee from the object (or remove the object from my knee) because the object is already gone. And if I do so, there will be no difference in tactile stimulation because the tactile signal has already stopped. More generally, it is difficult to imagine the interdependence of perception and action, when perception is so temporary that it does not afford the time to act. Most sensorimotor expectations require enduring perceptual experiences to make sense. If the object is no longer present, there cannot be any perceptual consequences. Hence, there cannot be any sensorimotor contingencies. If this is true, then 
there is no possible enactive account of instantaneous tactile experiences (or of any other kind of instantaneous perceptual experience).

The only way out is to include not only sensorimotor knowledge, but also mere motor knowledge in the enactive account. One may indeed argue that it does not matter that I am too late in removing my knee from the object that briefly touched me. The action does not even need to be performed; it merely needs to be triggered by the tactile signal. The urge to move would constitute the perceptual experience, independently of its bodily execution, its sensory feedback, and the remaining presence of the sensory input that elicited it. Procedural knowledge does not require that the object that it applies to still exists. Let us imagine that you are telling me about a restaurant that you really used to like, which closed recently. If I ask you where the restaurant was, you may still know how to get there (although the restaurant is no longer there). Similarly, I can still act toward the location of the tactile stimulus, although the stimulus is no longer available. Feeling that I was briefly touched on the knee is for me knowing how to get to where I was touched. ${ }^{6}$ On this view, the spatial content of the tactile experience that I was touched on the knee is determined by the procedural knowledge of how to get to the bodily location of the tactile stimulation. Hereafter I shall refer to this type of procedural knowledge as 'spatial know-how'. Accounts in terms of spatial know-how sound like a plausible working hypothesis for the enactive approach for two reasons. ${ }^{7}$ On the one hand, this kind of procedural knowledge seems to be consistent with the

\footnotetext{
${ }^{6}$ If I reach the body location that was touched and put my finger on it, then I shall receive tactile signals from the same place. In this latter case, there would be tactile consequences.

${ }^{7}$ However, one may argue that the spatial content of bodily experiences must be determined by the movements performed by the specific body part that is experienced. For instance, the tactile experience of my knee being touched should be determined by the movements of my knee only. On the contrary, pointing and reaching movements are performed with a body part that is not represented in the tactile content, namely, the hand. Should this type of movement be discounted for this reason? This would be too restrictive. Most of the actions that one can perform towards one's body are hand movements, and if one discounts them as irrelevant, then there are not many movements that are left over. Furthermore, there are some body parts that are not movable, and yet, we can experience them being touched. Therefore, one might suggest that the spatial content
} 
enactive view ("What we perceive is determined by (...) what we know how to do" Noë, 2004). On the other hand, I do not see any alternative type of procedural knowledge that could constitute instantaneous passive touch. ${ }^{8}$

To sum up, the link between touch and bodily activity is supposed to be so obvious that it does not deserve a proper investigation and it should help us to better understand the enactive view of vision. However, this might be true for haptic touch only, and only for the exteroceptive content. The enactivists still need to give a specific account of the relationship between tactile experiences and action in the case of instantaneous passive touch, and this is not an easy task. They may argue that it is precisely because the relation with action is so weak that the content of such tactile experiences is so poor. That might be true. Nonetheless, for the enactive account to be true, there needs to be at least one type of law-like relation with action if there is to be tactile experience at all. I suggest that the most plausible way to spell out an enactive account of tactile content is in terms of reaching movements made toward the bodily location that was touched. However, we shall see now that this view faces severe difficulties when confronted with the empirical literature on bodily experiences. Instead of the interdependence of action and tactile experiences, I shall show that they are independent, and that they can be dissociated in both pathological and normal cases.

\section{Dissociating bodily experiences and spatial know-how}

of the tactile experience depends on the procedural knowledge of the body part that is touched with respect to the rest of the body.

${ }^{8}$ The enactive view in terms of spatial know-how may look close to Anscombe's dispositional theory of bodily sensations. The difference, however, is that the enactive view aims at accounting for the spatial content of bodily experiences. By contrast, Anscombe assumes that bodily sensations are not intrinsically spatial, and as such, they cannot have any spatial content. Yet, some of the arguments presented here can be seen as objections against Anscombe's view as well. 
One of the most recurrent and powerful empirical counterarguments provided against the enactive approach to vision comes from the neuropsychological dissociations between optic ataxia and visual agnosia. ${ }^{9}$ For example, the visual agnostic patient DF was presented with a set of various squares and rectangles. She was at chance when required to match the width of such simple geometrical forms by scaling the distance between her thumb and index finger (i.e. manual, non-verbal report). ${ }^{10}$ By contrast, she was accurately able to grasp the blocks. The reverse pattern of performance was found in optic ataxic patients. The conclusion drawn from such double dissociation is that one can have visual experiences of an object without being able to act towards this object, and vice-versa. That is, visual awareness can operate independently of visually-guided actions. A similar line of evidence against the enactive approach to vision comes from healthy individuals. It was found that action can be immune to visual illusions, such as the Müller-Lyer illusion, the Ponzo illusion, the Titchener illusion or the Hollow Face illusion. For example, the Hollow Face illusion is a visual illusion, in which the perception of a concave (or hollow) mask of a face appears as a normal convex (or protruding) face. It was found that if asked to quickly flick a magnet off the nose (as if it were a small insect), participants did not direct their finger movements to where the nose appeared to them (i.e. the protruding nose). They directed them to the actual location of the target in the hollow face, which was 8 inches away from the apparent location. In other words, the content of the visual experience of the face does not correspond to the visually-guided movements

\footnotetext{
${ }^{9}$ For a comprehensive review of the empirical literature that supports what is called the Perception-Action model of vision, see David Milner and Mel Goodale, The Visual Brain in Action. Oxford: Oxford University Press, 1995 and Pierre Jacob and Marc Jeannerod, Ways of Seeing, the Scope and Limits of Visual Cognition Oxford: Oxford University Press, 2003. For an extensive discussion of the relevance of this type of empirical evidence for the enactive approach, see Pierre Jacob (2008), Ned Block, "Review of Alva Noë, Action in Perception”, The Journal of Philosophy, CII (2005): 259-272; Andy Clark, Supersizing the mind. New York: Oxford University Press, 2008.

${ }^{10}$ Some hand movements can count as perceptual reports. For instance, it was shown that the underlying processes of scaling the distance between thumb and index finger are very different, whether it is for reporting a perceptual judgment or for a visuomotor task of grasping (Marc Jeannerod, The Cognitive Neuroscience of Action. Oxford: Blackwell, 1997).
} 
directed toward the face. This was taken as evidence that the content of visual experiences (e.g., convex face) is at variance with the content of action-oriented vision (e.g., hollow face). What we see is not determined by what we do, or what we know how to do.

Is there any equivalent type of empirical evidence against the enactive view of bodily experiences? The reply is yes. ${ }^{11}$ On the basis of dissociations between tactile and proprioceptive experiences on the one hand, and spatial know-how on the other hand, both in patients and in healthy individuals, I shall argue against the enactive view of (i) the phenomenology of bodily sensations, and (ii) the spatial content of bodily experiences.

\subsection{Action without sensation}

A patient is amazed at her own ability while blindfolded to point to where she was touched on her hand while she felt absolutely no sensation of having been touched (see introduction). This patient suffers from what is called 'numbsense' in the neuropsychological literature (also called 'blind touch'). Following cortical or subcortical lesions, patients with numbsense become completely anaesthetized on their right side. They have a complete lack of tactile experience. They are not able to detect, localize and describe tactile stimuli on their right arm, nor are they able to indicate on a picture of an arm where the stimulus was applied, even in a verbal forced choice condition. What is interesting is that despite their apparent numbness, they are able to accurately guide their opposite hand toward the specific site where they were touched when so instructed, and to their own surprise. Their bodily movements did not even improve their performance in verbal localization. When patients were asked both at

\footnotetext{
${ }^{11}$ For numbsense patients, see Paillard, Michel and Stelmach (1983) as well as Y. Rossetti, G. Rode and D. Boisson, "Implicit processing of somaesthetic information: a dissociation between where and how?" Neuroreport. 6 (1995): 506-10. For a description of KE and JO, see H.A. Anema, M.J. van Zandvoort, E.H. de Haan, L.J. Kappelle, P.L. de Kort, B.P. Jansen, H.C. Dijkerman, “A double dissociation between somatosensory processing for perception and action”. Neuropsychologia. 47 (2009):1615-20.
} 
the same time to verbally localize and to point to where they had been touched, they indeed became equally bad. In addition, they may be unaware of their arm location, and yet be able to accurately reach the position of their arm. Interestingly, this type of dissociation was known to Merleau-Ponty. He described the case of the patient Schneider who was unable to localize where a mosquito was stinging him, and yet, was able to scratch his leg where he was stung.

When numbsense patients scratch their leg where they were stung, they must have mastered sensorimotor information about reaching their leg, scratching it and the effects of these actions. Yet, despite this sensorimotor knowledge, they feel nothing. Here, we have a clear case of a complete deficit of tactile and proprioceptive phenomenology with preserved spatial know-how. The case of numbsense therefore shows that spatial know-how (and its exercise) per se is not a sufficient condition of tactile and proprioceptive phenomenology.

\subsection{Action without perception, perception without action}

As said earlier, some versions of enactivism aim at explaining how perceptual experiences come to represent spatial properties, rather than explaining how they come to have a conscious phenomenology. Hence, it might be argued that the evidence provided by the numbsense case is of no relevance for these versions of the enactive view. More challenging for them is the following case of the patients $\mathrm{KE}$ and $\mathrm{JO}$, who have tactile experiences, but whose tactile experiences are dissociated from spatial know-how, in the same way as visual experiences in optic ataxia and visual agnosia.

KE and JO can both consciously feel a touch. In addition, they can accurately report the location of a visual target and point to it. Yet, when asked to point to where they were touched, they displayed a surprising double dissociation. They were asked either to point to 
where they were touched on the hand (i.e. tactile-motor task) or to point to the location of the tactile stimulus on a pictorial map of their hand (i.e. abstract and detached perceptual report). It was found that JO failed to point accurately to the hand map, whereas she did not fail to point accurately to her own hand. Conversely, KE failed to point accurately to his own hand, but not to the hand map.

In both cases, the spatial content of the tactile experiences does not match with the possession (or the deficit) of spatial know-how. JO experiences that she is touched, but she cannot report where she is touched. She merely experiences the touch 'somewhere', with no determinate spatial content. Yet, like numbsense patients, she is able to accurately get to the location of the touch on her own body. Hence, her spatial know-how does not suffice for providing her tactile experience with spatial content. By contrast, KE experiences and reports that he was touched on the hand in a specific location, but he is unable to get to the location of the touch on his own hand. Hence, there is no spatial know-how that could provide the spatial content of his tactile experience, and the tactile spatial content must have a different ground. ${ }^{12}$ The case of JO shows that spatial know-how per se is not a sufficient condition of tactile spatial content, whereas the case of KE shows that it is not a necessary condition.

To conclude, these dissociations show that the spatial information in the sensorimotor system can differ from the spatial information in the perceptual system. This leads us to infer that the sensorimotor system and the perceptual system cannot be reduced to one another. They are two distinct systems, one involved in action and the other involved in perceptual

\footnotetext{
${ }^{12}$ An enactivist may reply that there is no evidence of a deficit in spatial know-how in KE. There is only evidence of a deficit in spatial motor performance, and this does not systematically reveal a deficit in competence. If so, tactile spatial content could still be accounted for in terms of the supposedly preserved spatial know-how. However, this explanation of the results does not sound plausible. The patient KE has no motor deficit, as shown by his preserved ability to point to a hand map or to neutral visual targets. His results can neither be explained by a proprioceptive nor a memory deficit. In the study, the patients' hands were moved passively to a different location and they were asked after two seconds to return their arms to the previously held position. KE's performance in this proprioceptive task was similar to JO's performance. It thus seems plausible to assume a deficit of spatial know-how in KE, which explains his poor motor performance when pointing to his own hand.
} 
experiences. The dissociation between the two types of spatial information specific to each system can be made even more salient in the context of bodily illusions in healthy individuals.

\subsection{Illusory spatial content}

If spatial know-how provides the spatial content of perceptual experiences, then it should explain why this content can become illusory in healthy subjects. However, as said before, it was found that action can be immune to visual illusions. We shall see now that bodily illusions as well cannot be explained by mistaken information encoded in spatial know-how. I shall describe here the Rubber Hand Illusion, although similar results have been found in the vibrotactile illusion (i.e. the illusory kinaesthetic experience that one's arm is stretching induced by biceps tendon vibration). ${ }^{13}$

In the Rubber Hand Illusion (RHI), participants sit with their left arm resting on a table, hidden behind a screen. They are asked to fixate on a rubber hand presented in front of them, and the experimenter simultaneously strokes both the participant's hand and the fake hand with two paintbrushes. After a short while, the majority of participants report that they feel the touch of the paintbrush in the location where they see the rubber hand touched. Even more surprisingly, they may feel as if the rubber hand were their own hand. At the behavioural level, participants report their hand as closer to the rubber hand than it really is. This is true only when the two hands are synchronously stimulated and in congruent positions.

\footnotetext{
${ }^{13}$ For a description of the standard Rubber Hand Illusion, see M. Botvinick and J. Cohen, "Rubber hands 'feel' touch that eyes see". Nature. 391 (1998): 756. For a description of the standard vibrotactile illusion, see J.R. Lackner and P.A. DiZio, "Aspects of body self-calibration". Trends Cogn Sci. 4 (2000): 279-88. The immunity of action to the vibrotactile illusion was shown by M.P. Kammers, I.J. van der Ham and H.C. Dijkerman, "Dissociating body representations in healthy individuals: differential effects of a kinaesthetic illusion on perception and action". Neuropsychologia. 44 (2006): 2430-2436. The immunity of action to the Rubber Hand Illusion was shown by M.P. Kammers, F. de Vignemont, L. Verhagen and H.C. Dijkerman, "The rubber hand illusion in action". Neuropsychologia. 47 (2009): 204-11.
} 
Now, the enactive view might account for the illusion in terms of erroneous spatial knowhow. The explanation may run as follows: participants feel their hand closer to the rubber hand because they were misleadingly induced to expect that if they reach the location close to the rubber hand, they will touch their own hand. However, a recent experiment refutes this explanation of the RHI. It was found that participants accurately directed their opposite hand to the real location of their own hand that was touched, and not to the illusory location that they reported. Their reaching movements were not sensitive to the spatial illusion. Similarly, when they performed the reverse movement (i.e. directing their touched hand toward their opposite hand), they had the right know-how of where their hand was. The complete absence of illusion was also confirmed in a bimanual task, where participants had to grasp a stick in front of them. Again, their bodily movements revealed accurate spatial information about the relationship between their two hands as well as the use of spatial know-how of their correct locations. When participants were asked a second time to make a perceptual judgment about the location of their touched hand after having moved, they were still sensitive to the RHI and they still experienced their hand as being closer to the rubber hand than it was.

Although participants experienced their hand as being closer to the rubber hand than it was, they knew how to reach it and they knew where it was when asked to move it. Hence, the RHI cannot be explained by inaccurate spatial know-how. The illusory spatial content of the bodily experience is not determined by the illusion-immune spatial know-how, which reveals an accurate sense of the position of the touched hand.

To sum up, in healthy individuals, like in patients, the spatial content of tactile and proprioceptive experiences can be dissociated from the spatial information encoded in spatial know-how used to guide reaching and pointing movements, such that one can be inaccurate in one and not the other, and vice-versa. Numbsense patients even illustrate the possibility of preserved know-how with no associated tactile sensation. Hence, it seems that spatial know- 
how, as recruited by reaching, pointing and grasping movements, is neither necessary nor sufficient for bodily experiences.

\section{The dilemma}

In touch, like in vision, these various cases raise interesting questions for the enactive approach. It seems that at this stage, the enactive approach faces a dilemma. In a nutshell, if the enactive account of bodily experiences is in terms of spatial know-how, then it is false, because spatial know-how is separable from bodily experiences. If the enactive account is not in terms of spatial know-how, then it is unclear what type of sensorimotor expectancies could provide both the exteroceptive and the interoceptive spatial content of any kind of tactile experiences, including instantaneous passive touch. Let me briefly develop the two horns of the dilemma.

On the first horn of the dilemma, the proponents of the enactive approach characterize the spatial content of tactile experiences in terms of the spatial know-how relative to the bodily location that has been touched (how to reach it or how to move it). But we have seen that this enactive account is challenged by a series of empirical results that reveal that contrary to what the enactive approach predicts, tactile experiences are separable from the perceiver's actions. On the one hand, the possession of accurate spatial know-how does not guarantee that one consciously feels the touch (e.g., numbsense). Nor does it guarantee that the spatial content of bodily experiences is accurate (e.g., patient JO; Rubber Hand Illusion). On the other hand, the lack of spatial know-how does not guarantee that one has no bodily experience, nor that the bodily experience has no spatial content (e.g., patient KE). These results are difficult to accommodate for the enactive approach if it aims at explaining tactile experiences in terms of the procedural knowledge that is involved in reaching, pointing or scratching one's body. What we feel is not determined by what we do, nor by what we know how to do. 
On the second horn of the dilemma, the proponents of the enactive approach discount spatial know-how involved in such movements as irrelevant for an enactive account of bodily experiences. On this view, they claim to be interested in what perceptual experiences are grounded in, and not in what they are for. ${ }^{14}$ Hence, they reject these movements as mere "practical consequences" of the bodily experiences. The link between perception and such types of action is said to be only instrumental. It is thus no surprise if this type of spatial know-how is independent and dissociable from bodily experiences. However, if the proponents of the enactive view want to argue that spatial know-how does not involve the right kind of sensorimotor knowledge for bodily experiences, then they have to face several difficulties.

First, they must explain why this type of relationship between perception and action is merely instrumental, rather than constitutive. Why couldn't perceptual experiences be grounded in what they are for? It is unclear on what grounds the line between the 'right' type of procedural knowledge and the 'wrong' type of procedural knowledge is drawn. As already mentioned, an enactivist may reject reaching and pointing movements because they do not affect the tactile content in the same way that turning my head affects the visual content. However, this may be true of reaching, pointing and grasping movements, but not of scratching where there is a clear corresponding sensory consequence of one's movements (e.g., if one scratches where one has been bitten, the stimulation decreases). Yet, we have seen that Merleau-Ponty's patient Schneider had no conscious experience of the itching, although he could accurately scratch his leg. If this type of sensorimotor contingency is not included in the set of patterns of interdependence between stimulation and movement that constitutes itching experiences, then it is hard to see what would be. On the other hand, it is not clear that

\footnotetext{
14 For this line of argument, see for instance Alva Noë, "Vision without representation". In N. Gangopadhyay, M. Madary, and F. Spicer (eds), Perception, Action and Consciousness, Oxford: Oxford University Press (in press).
} 
enactivism can relinquish procedural knowledge that cannot be articulated in sensori-motor terms. Take the example of the experience of a flash of light. What is the enactive account for the temporal content of the visual experience (e.g., experience of a brief flash)? There is no corresponding sensory expectation of how one's movements would affect the flash of light, given that the flash already belongs to the past. Furthermore, one cannot appeal to a counterfactual of the type "if the visual object were still here and if I did $x$, then it would have the visual consequence $y$ ". Indeed this would not account for the brevity of the visual stimulus. Hence enactive explanations must sometimes accept procedural knowledge with no possible modification of the sensory signal. And if this is true for temporal content, why not for spatial content as well? Especially since the enactivist is left empty handed with no sensorimotor account of the spatial content of bodily experiences if she discounts spatial know-how. As I said in the beginning, it is highly unclear what sensorimotor contingencies are supposed to be in the case of passive touch, especially if the touch is very brief. One cannot simply highlight the importance of action for bodily experiences; one has to give a detailed account of the specific set of non-instrumental sensorimotor expectations that constitute bodily experiences. If the type of procedural knowledge that I offered here is not viewed as relevant for the enactive approach, then I would like to invite the proponents of such an approach to provide an alternative, which should be empirically testable.

However, that might be an impossible task. As we have seen, the discounting of reaching, pointing, grasping and scratching movements supposes distinguishing between two types of procedural knowledge (constitutive and instrumental), and thus distinguishing between two distinct action systems, the action system that is constitutively linked to perceptual experiences and the action system that is not. If there are such dual sensorimotor systems, it should be possible to dissociate them, but one may wonder whether one sensorimotor system can be impaired while the other is preserved, or vice-versa. Alternatively, the enactivist has to 
postulate that there is a unique action system, but that it exploits two distinct sets of spatial information about the location of the sensation, one of them being identical to the spatial content of bodily experiences. ${ }^{15}$ For instance, in the RHI, the location of the hand that was stroked would be encoded as $2 \mathrm{~cm}$ farther for one type of action than for another. The spatial information exploited by the motor system would thus not be internally consistent. This seems highly unlikely. As far as I know, there is no empirical validation of such a dissociation within the motor system. Any sensorimotor account of the specific location of a sensation has to involve an action that reveals a pinpointing of that location in the sensorimotor system. And it is unlikely that there can be two distinct sensorimotor systems, and not even two distinct sets of spatial information exploited by the sensorimotor system.

To summarize, either the proponents of the enactive approach claim that bodily experiences are determined by spatial know-how involved in reaching and pointing movements or they do not make such a claim. If they do, then the version of the enactive account of bodily experiences that has the most plausibility is subject to the concerns I raised in this paper. If they do not, then the burden of proof is on the enactive side to distinguish between the sensorimotor knowledge that does and does not constitute bodily experience and to propose an alternative sensorimotor account of bodily experiences, which involves assuming the existence of two sensorimotor systems or of two distinct sets of spatial information exploited by the sensorimotor system. The enactive approach to bodily experiences thus suffers from serious flaws. And if the enactive approach does not work for bodily experiences, which seem at first sight the less controversial example, then it is even less plausible that it works for visual experiences.

\footnotetext{
15 There could be two different ways of presenting the same spatial information, for instance within two distinct frames of reference. But this is different from two different sets of spatial information.
} 
It has been suggested that vision evolved in the first place, not to provide conscious perceptual experiences, but to provide distal sensory control of movements. It is only later in evolution with the emergence of more and more complex behaviours that vision evolved to provide internal models of the world, stored in memory and accessible to other cognitive systems. Perception and action require different transformations of the visual signal. For instance, action requires on-line adjustments, whereas perception, which allows us to recognize objects, is concerned with enduring visual properties. In addition, action requires the object one acts upon to be localized relative to oneself within an egocentric frame of reference, whereas there is no such requirement for perception, which is able to make allocentric judgments on the relative locations of two objects. These distinctions result in the evolution of two visual systems, which most of the time interact, but are dissociable.

Similarly, it seems that bodily information processing evolved to be used first for action, and only later for admiring oneself in the mirror. What is needed for action is different in many respects from what is needed for bodily experiences. As such, the very same bodily information is encoded in two different formats, and only one of them is linked to action. For instance, what is needed for action is constantly updated bodily information such that moment to moment one knows the posture of one's body parts. By contrast, we have seen in the RHI study that participants still experience their hand as closer to the rubber hand after having moved it. This shows that their bodily experience had not been updated by proprioceptive information. These findings, among others, suggest that there are two functionally defined somatosensory systems, which interact most of the time, but are dissociable. One may further suggest that they lead to two distinct functionally defined representations of the body, the body schema for action (i.e. information about the body necessary to move such as posture, limb size, and strength) and the body image for perception (i.e. judgment of one's own bodily properties). The dissociations in touch and proprioception show that vision is not an exception 
in the architecture of the mind. Other modalities, including touch and proprioception, use a dual coding based on the functional distinction between perception and action. The brain encodes information in distinct formats and the way it uses information determines the way it encodes it. ${ }^{16}$

\footnotetext{
${ }^{16}$ I am grateful to Ned Block and Pierre Jacob for their useful comments and their support.
} 